\title{
Experiências de mulheres vivendo com HIV gestantes ou lactantes num contexto de Moçambique: revisão integrativa
}

\author{
Experiences of pregnant or lactating \\ women living with HIV in a Mozambican \\ context: an integrative review
}

\section{Bento Saloio Daniel Mazuze ${ }^{1}$ (1) Thayna Davi de Souza Borges 2 (1) Luiza Tavares Maciel Rodrigues Brasil ${ }^{3}$ (1) Larissa Polejack ${ }^{4}$ (1)}

\begin{abstract}
1Autor para correspondência. Universidade Eduardo Mondlane (Maputo). Moçambique. loymz@yahoo.com.br 2-4Universidade de Brasília (Brasília). Distrito Federal, Brasil. thayna-davi@hotmail.com, luizatmrbrasil@gmail.com, larissapolejack@hotmail.com
\end{abstract}

\begin{abstract}
RESUMO | OBJETIVO: Conhecer e documentar as experiências e as principais dificuldades vivenciadas pelas gestantes ou lactantes soropositivas. MÉTODO: Revisão integrativa da literatura com busca de produções sobre as experiências e principais dificuldades enfrentadas por mulheres vivendo com HIV/Aids, gestantes ou lactantes nas bases de dados BVS, periódicos da CAPES e SciELO, realizada em março de 2021. RESULTADOS: Foram identificadas 256 pesquisas, publicadas no período de 2016 a 2021. Destacou-se que dos 256; 70 resumos são da BVS, 181 resumos dos periódicos CAPES e 5 resumos são da SciELO; apenas 18 artigos publicados nos últimos 5 anos discutem a temática em causa. CONCLUSÃo: As muIheres HIV positivas gestantes ou lactantes podem vivenciar um sofrimento psíquico caracterizado pelo medo de infectar a criança durante a gestação, no parto ou durante a amamentação; referem ainda o receio de partilhar o diagnóstico de HIV positiva enquanto gestantes ou lactantes à família, e amigos para evitar a discriminação e estigmatização perante a sociedade que ainda não aceita que a mulher HIV positiva também tem o direito de ser mãe.
\end{abstract}

PALAVRAS-CHAVE: Gestação. Amamentação. HIV/AIDS. Transmissão vertical e saúde mental.

\begin{abstract}
OBJECTIVE: Know and document the experiences and main difficulties experienced by HIV-positive pregnant or lactating women. METHOD: integrative literature review searching for productions on the experiences and main difficulties women face with HIV/AIDS, pregnant or lactating in the BVS databases, CAPEs, and SciELO periodicals, carried out in March 2021. RESULTS: there were identified 256 types of research published in the period from 2016 to 2021. It is highlighted that of the 256, 70 are BVS's researches, 181 are CAPES's researches, and 5 are SciELO's summaries; only 18 papers published in the past five years discussed the subject matter. CONCLUSION: The HIV positive pregnant or lactating women may experience psychic suffering characterized by the fear of infecting the children during pregnancy, on labor, or during breastfeeding; they also refer to be afraid of sharing the diagnosis of HIV-positive while pregnant or lactating to their family and friends to avoid the discrimination and stigmatization before the society that does not yet accept that HIV-positive women have the right to the motherhood.
\end{abstract}

KEYWORDS: Pregnancy. Breastfeeding. HIV/AIDS. Vertical transmission and mental health. 


\section{Introdução}

No presente estudo, é abordada e problematizada a experiência e as dificuldades encaradas pelas muIheres HIV positivas gestantes e lactantes e os efeitos dessa vivencia para a sua saúde mental.

De acordo com o Programa Conjunto das Nações Unidas sobre HIV/Aids (UNAIDS), cerca de 1,7 milhão de pessoas em todo o mundo foram infectadas pelo vírus da imunodeficiência humana (HIV) e estes dados são referentes às últimas atualizações da UNAIDS do período entre 2010 a 2018. No entanto, houve progresso na África oriental e meridional, onde as novas infecções por HIV diminuíram 38\% desde 2010. Isso contrasta fortemente com a Europa oriental e a Ásia Central, que viram um aumento significativo de $72 \%$ em novas infecções por HIV desde 2010. As novas infecções por HIV também aumentaram no Oriente Médio e no Norte da África, em 22\%, e em 21\% na América Latina (UNAIDS, 2018).

A África Subsaariana concentra $80 \%$ do total das infecções por Tuberculose (TB) associada ao HIV no mundo, e as mulheres dessa região continuam sendo afetadas de forma desproporcional pela epidemia do HIV/aids em comparação com os homens. Na Zâmbia, por exemplo, a prevalência de infecção pelo HIV entre as mulheres com idades entre 15 e 24 anos é quase quatro vezes superior à dos homens do mesmo grupo etário (UNAIDS, 2018).

Nesse contexto, as mulheres que vivem com HIV/aids podem se deparar com desafios que incluem o sentimento de solidão, as dificuldades em falar com os outros sobre sua condição sorológica (mesmo para o companheiro ou para os filhos), ou ainda, com a insegurança e medo de perder amigos, familiares ou seus filhos. Na sociedade, na comunidade e no seu local de trabalho, as atitudes de discriminação também acontecem, o que acaba aumentando a possibilidade de haver sofrimento psíquico por parte da mulher HIV positiva (Padoin et al., 2010).

De acordo com Joint United Nations Programme on HIV/ AIDS (UNAIDS, 2018), nos últimos anos foram alcançados consideráveis avanços no combate ao HIV nas regiões leste e sul do continente africano, as quais concentram mais da metade (53\%) dos 36,9 milhões de pessoas que viviam com HIV no mundo em 2019. Em contrapartida, as regiões da África Subsaariana, Europa Oriental e Ásia Central registraram pouco progresso na redução anual de novas infecções por HIV, que dobraram a partir do ano 2000. Muito embora uma ampliação do acesso ao tratamento ocorrido nos últimos anos seja um aspecto positivo, abrangendo $68 \%$ dos adultos e 53\% das crianças (Organização Pan-Americana de Saúde, 2021; Organização Mundial de Saúde, 2021), as novas infecções pelo HIV diminuíram apenas $18 \%$ desde 2010 , bem menos que os $75 \%$ esperados até 2020 (UNAIDS, 2018).

No mundo, cerca de 16 milhões de mulheres vivem com o HIV, sendo muitas delas em idade reprodutiva. Estima-se que a taxa de transmissão vertical (TV) seja de $31 \%$, devido à ausência de intervenção terapêutica precoce. Metade do total das crianças menores de 15 anos existentes na África correm o risco de serem infectadas por meio da TV, sendo que $90 \%$ dessas infecções ocorrem na África Subsaariana (Friedrich et al, 2016).

De acordo com a UNAIDS (2020), dos oito países com mais pessoas infectadas pelo HIV no continente africano, o que apresenta o maior índice é a África do Sul, cujas novas infecções, em 2018, somaram 240.000 casos. O segundo país mais afetado é Moçambique (150.000 novas infecções), seguido da Tanzânia (72.000), Uganda (53.000), Zâmbia (48.000), Quénia (46.000), Malawi (38.000) e Zimbábue (38.000).

Moçambique possui uma das maiores taxas de infecção pelo vírus da imunodeficiência humana (HIV) no mundo. A prevalência de HIV na população de 15-49 anos é de 13,2\% (Ministério da Saúde de Moçambique, 2019), o que representa um recrudescimento da epidemia no país na última década, já que em 2009 o índice era $11,5 \%$.

\section{Transmissão vertical do HIV}

O vírus da imunodeficiência humana tipo 1 (HIV-1) pode ser transmitido da mãe para o filho durante a gestação, no momento do parto e através do leite materno. O risco de transmissão vertical do HIV, sem que ocorra qualquer intervenção durante a gestação para evitá-lo, situa-se entre $25 \%$ e $30 \%$. Desse percentual, o risco de transmissão intraútero é de $25 \%$ a $40 \%$ e intraparto de $60 \%$ a $75 \%$. A amamentação está associada a um risco adicional de transmissão, podendo chegar a $29 \%$ nos casos de infecção aguda. No recém-nascido, as portas de entrada do vírus são as mucosas da nasofaringe e do trato gastrintestinal (Sociedade Brasileira de Pediatria [SBP], 2017). 
Acabar com a epidemia de aids entre crianças, adolescentes e mulheres jovens requer metas ambiciosas e uma aceleração ainda mais intensa na resposta de combate à epidemia. Até 2016, estimava-se que 2,1 milhões de crianças viviam com HIV (UNAIDS, 2018).

Em 2016, o mundo registrou 160 mil novas infecções por HIV entre crianças, estando quase a sua totalidade (140 mil) concentrada nos 23 países mais atingidos pela doença no globo. No mesmo ano, cerca de 200 mil adolescentes entre 15 e 19 anos foram infectados pelo HIV, dos quais $72 \%$ eram mulheres jovens, levantando preocupações relacionadas a novas infecções por HIV entre mulheres jovens e meninas. Mais de $70 \%$ de todas as novas infecções por HIV entre jovens aconteceram na África Subsaariana, que tem a população jovem que mais cresce no mundo. Moçambique faz parte desta região, a qual apresenta as maiores taxas de infecção no mundo. Além disso, o país apresenta um elevado número de novas infecções por HIV entre mulheres adolescentes e jovens na população moçambicana, o que pode provocar uma explosão no número de afetados pelo HIV no futuro para toda a sociedade (UNAIDS, 2018).

As taxas de TV por HIV variavam no intervalo de 15$25 \%$ em crianças alimentadas com fórmula exclusiva, que é um composto lácteo artificial, e de $25-40 \%$ naqueles amamentadas ao seio. Com a introdução da terapia antirretroviral combinada (TARC) para as gestantes, ao final da década de 90 , houve uma redução de 20 vezes nas taxas de TV. O início precoce na gestação, a cesariana eletiva, a profilaxia medicamentosa perinatal e a prática de não-aleitamento (em países onde esta é possível e sustentável), contribuíram para a redução de até $95 \%$ da TV em países ricos, levando a taxas inferiores a 2\% (Friedrich et al., 2016).

Tais políticas de saúde pública que foram implementadas em vários países, como Brasil e Cuba, constituem a prova inequívoca de que a amamentação realizada por mães soropositivas, se interrompida precocemente, pode evitar a TV de HIV da progenitora para o(a) filho(a). Contudo, em Moçambique, por se tratar de um país em vias de desenvolvimento, e que ainda apresenta casos de desnutrição em recémnascidos, o Ministério de Saúde local ainda defende a política de amamentação até os dois anos de idade, o que pode ser um fator de risco para a TV, em especial se considerado o acesso à assistência médica e medicamentosa que a gestante infectada pelo HIV possui na região em que vive.
Segundo Friedrich et al. (2016) apresentaram em seus estudos, existe uma redução de taxas de TV para $1 \%$, quando a carga viral materna é suprimida a níveis indetectáveis durante a gestação. Em 2015, Cuba foi o primeiro país a receber a validação da OMS em relação à eliminação da TV do HIV, definida como menos de 50 casos para 100.000 nascidos vivos, com uma transmissão inferior a $5 \%$ em lactentes amamentados ao seio, ou inferior a $2 \%$ naqueles não amamentados, além do uso de ARV por mais de 95\% das gestantes.

Em países onde não há recursos para se suspender a amamentação de bebês expostos ao HIV, estudos como de Friedrich et al. (2016) demonstraram que o uso de antirretrovirais (ARV) por lactantes é seguro e reduz efetivamente a transmissão através do leite materno. Assim, o risco de transmissão pós-natal do HIV pode ser reduzido para menos de $2 \%$, nível que permite contemplar a prevenção da TV mesmo em locais onde há recursos limitados. A TARc durante a gestação é a intervenção mais efetiva para a prevenção da TV. Os ARV iniciados antes mesmo da concepção e mantidos durante toda a gestação resultam em taxas de transmissão extremamente baixas.

Moçambique é um dos países em que ainda não é possível suspender a amamentação quando o bebê está exposto ao HIV, devido a fatores como aspectos de ordem cultural em torno da amamentação, estigma social relacionado a interrupção precoce da amamentação e os problemas de acesso aos serviços de atendimento básico durante a gestação. Assim, a TV ainda constitui um fator de risco e pode impactar o estado emocional da mãe soropositiva durante a gestação.

Esse cenário não é a realidade da maioria dos países que convivem com a epidemia de HIV/aids. A Organização Mundial da Saúde (OMS) revela que nos 22 países onde mais de $90 \%$ das gestantes estão expostas ao HIV, apenas $44 \%$ das mesmas foram testadas para HIV. Das que sabidamente convivem com a doença, $73 \%$ receberam ARV na gestação e $61 \%$ durante a amamentação. Apenas $50 \%$ das crianças expostas receberam profilaxia com ARV, e dessas, somente $44 \%$ foram testadas até os dois meses de vida. Outro índice preocupante é que apenas 32\% das crianças que foram diagnosticadas como infectadas estavam recebendo ARV adequadamente (Friedrich et al., 2016). 
De acordo com Hoffmann et al. (2014), para prevenir a TV, O TARV deve iniciar na fase precoce da gestação, especialmente para mulheres que requerem o uso de ARV para suas próprias indicações clínicas. No momento do parto, o tempo de exposição à ruptura de membranas também é importante. Além disso, a redução do parto prematuro por meio de um cuidado pré-natal adequado é eficaz na redução da TV intraparto e pós-natal precoce.

A pobreza extrema da mulher, somada à sua dependência familiar para acessar a assistência médica e medicamentosa, e a ausência de poder de decisão em relação à família que recai sobre a população feminina são aspectos cuja investigação mais aprofundada se vislumbra necessária. E o aprofundamento sobre as principais dificuldades vivenciadas pelas mulheres gestantes HIV positivas durante a gravidez ou amamentação, assim como dos seus parceiros sexuais, ainda é pertinente. Por isso, a revisão integrativa de literatura foi norteada pela seguinte questão: Quais são as experiências e principais dificuldades enfrentadas por mulheres vivendo com HIVIAids, seus parceiros e pelos profissionais de saúde que assistem os pacientes durante a gravidez ou amamentação?

Assim, o presente estudo possui como objetivo conhecer e documentar as experiências e as principais dificuldades vivenciadas pelas mulheres gestantes ou lactantes soropositivas.

\section{Metodologia}

Para a elaboração deste estudo, foi realizada uma revisão integrativa de literatura, seguindo os seguintes critérios:

i) Consulta às seguintes bases de dados: Biblioteca Virtual de Saúde - (BVS), Periódicos da Coordenação de Aperfeiçoamento de Pessoal do Nível Superior (CAPES) e Scientific Eletronic Library Online (SciELO). A escolha destas plataformas esteve relacionada a similaridades dos descritores usados na área da saúde no Brasil e em Moçambique.

ii) Consulta nas bases de dados das combinações entre os descritores com a utilização dos operadores booleanos: Experiência AND Gestação AND
HIV; Experiência AND Aleitamento materno $A N D$ HIV; Lactação AND Experiência AND HIV; HIV AND Gestação AND Aleitamento materno; Transmissão vertical AND Gestação AND Aleitamento materno; Transmissão vertical AND HIV AND Aleitamento materno; Saúde mental AND Mulheres AND HIV; Saúde mental $A N D$ Assistência integral em saúde $A N D$ HIV; Experiência $A N D$ Gestação AND HIV, e a consulta resultou em 256 artigos.

iii) Critérios de inclusão: (1) artigos que abordam as áreas temáticas em discussão no projeto de pesquisa nomeadamente: Mulher HIV positiva; gestante; lactante soropositiva; parceiro sexual; transmissão vertical e promoção de saúde ou saúde mental da mulher HIV positiva. (2) de 256 artigos, apenas 18 preenchiam os critérios previamente estabelecidos e serviram para a elaboração da revisão integrativa de literatura. Destes, nenhum artigo foi encontrado repetido nas diferentes plataformas. Foram lidos os resumos para analisar se o conteúdo estava de acordo com os objetivos do estudo e, em seguida, leu-se todos os artigos que satisfaziam a pesquisa.

Excepcionalmente, dois artigos que foram utilizados neste estudo não fazem parte do espaço temporal dos últimos cinco anos, mas possuem um conteúdo valioso para a sustentação cientifica de algumas informações apresentadas nesta pesquisa.

\section{Resultados e discussão}

Foram utilizados 18 artigos que abordavam a temática e os objetivos em questão, sendo eles (Alvarenga et al., 2019; Bastos et al., 2019; Bellotto et al., 2019; Bick et al., 2017; Friedrich et al., 2016; Mann \& Monteiro, 2018; Mindry et al., 2017; Ministério da Saúde, 2019; Pinto et al., 2017; Rodrigues et. al., 2020; Silva et al., 2020; Siqueira et al., 2020; UNAIDS, 2017; Vescovi et al., 2016). Para além dessas literaturas foram utilizados dois autores pela importante contribuição ao tema, sendo eles (Cartaxo et al., 2013; Zihlmann \& Alvarenga, 2015). A partir disso, discutimos 4 eixos, a gestação da mulher HIV positiva, as vivências da muIher com HIV diante do aleitamento materno, a (in) possibilidade de Transmissão vertical, e a Promoção da saúde da mulher HIV positivo. 


\section{A gestação da mulher HIV positiva}

No início da epidemia, na década 80, as mulheres infectadas pelo HIV eram desencorajadas a engravidar e fortemente censuradas pela equipe de saúde, o que resultava no aborto ou abandono do tratamento (Zihlmann \& Alvarenga, 2015). Contudo, apesar de atualmente os serviços de saúde atuarem adequadamente para a prevenção da Transmissão Vertical, ainda incorrem na desatenção nos cuidados que oferecem à mulher, pelo fato de persistir a histórica priorização da assistência focada na criança (Mindry et al., 2017).

Para entender a gestação sob a perspectiva de pessoas infectadas pelo HIV, é fundamental fazer uma análise mais clara das políticas públicas, pelo fato de abordarem de modo simplista e homogeneizante, não contemplando as singularidades e a complexidade da vivência com o vírus numa sociedade repleta de estigmas (Ministério da Saúde, 2019).

Em estudos de Silva et al. (2020), depois da descoberta da gestação, as mulheres expressaram a ambivalência de sentimentos com a notícia, referindo surpresa, nervosismo, tristeza e alegria. Apresentaram ainda, medo de vivenciar a gestação devido à sua infecção pelo HIV. Outras, minoritariamente, relataram apreensão devido à idade. E a constatação de uma gravidez no contexto de HIV em que, durante a experiência assistencial prévia, a possibilidade da Transmissão vertical não tenha sido minimamente abordada como fonte de reflexão e condutas decorrentes - parece ser potencialmente mais capaz de produzir repercussões negativas na saúde materna, além de favorecer a cogitação pelas gestantes da interrupção da gravidez por meio de um aborto induzido.

Para Vescovi et al. (2016), a descoberta do HIV durante a gravidez apresenta-se para as mulheres como um fator de risco, dentre outros motivos, pela intensa preocupação com a possibilidade de infecção do bebê e as eventuais complicações decorrentes da mesma. Um estudo realizado por Ingram e Hutchinson (2000) revelou também que as mães que tinham descoberto o HIV durante a gestação lembravam-se desse fato como algo especialmente difícil. Ethier et al. (2002); Kwalombota (2002); Pinto et al. (2017) exortaram para a necessidade de identificação dos riscos psicossociais, depressão e desordens somáticas envolven do o diagnóstico de infecção pelo HIV/aids durante o período gestacional.
A vigência do HIV durante a gravidez pode ser destacada como fator de risco para as mulheres, porque gerou a necessidade de acompanhamento médico especializado. A infecção dos companheiros criou um risco adicional, pois, ainda que o casal fosse soroconcordante, as orientações vigentes reforçam a importância do uso do preservativo nas relações sexuais, sobretudo para evitar reinfecções e aumento da carga viral de ambos (Vescovi et al., 2016).

A gestação na presença do HIV não necessariamente reduz os sentimentos positivos quanto à maternidade e ao filho, mas, certamente, impõe muitos temores e cuidados estritos para prevenir a Transmissão vertical, particularmente, no nascimento. As vivências de culpa e medo de transmitir do HIV para o filho (a) se associa à centralidade que as políticas e ações em saúde conferem à profilaxia. Apesar de assumir uma posição de obediência frente à profilaxia e à medicalização necessária, as mulheres manifestam desejos sobre o tipo de parto e sobre como queriam vivenciar esse momento. E entende-se que o diálogo deve ser fortalecido no sentido do vínculo e do apoio sem julgamentos às mulheres, emponderando-as quanto ao parto na presença do HIV (Bellotto et al., 2019).

Os aspectos psicológicos das mulheres que recebem resultado positivo para HIV na gestação podem ser profundamente afetados, sendo que a principal experiência vivida por essas mulheres está relacionada ao medo da transmissão ao feto, tornando esse medo uma parte importante de seu universo emocional, pois, em geral, o nascimento está associado a sentimentos positivos. Percebe-se, porém, que, quando existe a possibilidade de contaminação de seu bebê, a angústia ganha corpo e assume um papel importante no espectro psicológico dessas gestantes. Há a necessidade de orientá-las e redesenhar a subjetividade relacionada ao risco de contaminação de seus filhos pelo HIV. O processo gestacional pode então tender a ser uma experiência baseada em medos, trazendo tensão e insegurança, tanto em relação à transmissão do HIV, quanto no uso de medicamentos, temendo assim pelo sofrimento da criança em relação a todos os estressores aliados à Infecção por HIV. Ressalta-se que estar grávida na presença do HIV/AIDS, além das ambivalências sentimentais, faz com que a mulher tenha que se reconhecer como mãe e ter consciência de todas as consequências e riscos que essa condição acarreta (Bastos et al., 2019). 


\section{Vivências da mulher com HIV diante do aleita- mento materno}

O esforço para eliminar novas infecções pelo HIV em crianças tem sido global. Atualmente, 1.800 .000 menores de 15 anos vivem com HIV no mundo, o que representa menos de $1 \%$ do total de pessoas HIV positivas (UNAIDS, 2016).

O Brasil é um exemplo dos países que realizam o aconselhamento das mães que vivem com HIV sobre a não amamentação e uso da formula láctea infantil disponibilizada gratuitamente pelo Sistema Único de Saúde (SUS) e garantida pela Portaria GM/MS número 2.313 de 19 de dezembro de 2002 (Ministério da Saúde do Brasil, 2017). No entanto, em Moçambique não existe ainda a viabilidade da distribuição de formula para substituir o aleitamento materno, e o Ministério de Saúde local continua a orientar os profissionais de saúde para prosseguir com o mesmo até que a criança complete dois anos de idade, o que pode abrir espaço para o risco de transmissão vertical.

A mulher tem sobre si uma forte expectativa social relacionada à maternidade, na qual, a decisão de mãe, sendo HIV positiva, desvia-se da normalidade social e cultural. Para a sociedade, é como se estivesse a violar suas crenças, o que gera, para a mulher gestante, o medo da discriminação e rejeição, levando a pensar que as mulheres que vivem com HIV não possuem e não devem possuir vida sexual e reprodutiva ativa, chegando a culpá-las quando ocorre uma gravidez. É por isso que o significado em torno do aleitamento materno pode fazer com que as mulheres se questionassem sobre o vinculo mãe-filho. Essas mulheres acreditam que a amamentação possui um papel central na vinculação entre mãe e filho, e podem associar a alimentação artificial a dificuldades de interação e eventual prejuízo no desenvolvimento da criança (Cartaxo et al., 2013; Alvarenga et al., 2019). Quanto à saúde geral do bebê, as mães relataram apreensões e a vivencia de sentimentos de medo e incerteza.

Para Alvarenga et al. (2019), os discursos sociais e simbólicos em torno da amamentação parecem influenciar o sofrimento materno. $O$ discurso de que o leite materno é o melhor alimento para o bebê é reproduzido pelas mulheres, o que potencializa a tristeza e influencia no provimento de uma alimentação por elas considerada mais adequada para a criança.
As mulheres podem vivenciar isolamento, insegurança, sofrimento, por vergonha de que alguém descubra seu diagnóstico de soro positividade, e por medo de sofrerem preconceitos, discriminação e indiferença por parte dos que estão a seu redor, como a família, companheiro e amigos (Rodriguez et al., 2014).

Evidencia-se, na abordagem da mulher que vive com HIV, o medo de transmitir o vírus para o filho durante a amamentação, pois a possibilidade da infecção do filho está diretamente ligada ao sofrimento e ao preconceito que a doença traz consigo ao adquirir o vírus.

\section{A (in) possibilidade de Transmissão vertical}

A transmissão vertical (TV) do HIV é um evento multifatorial, com fatores de risco e de proteção, incluindo principalmente as características maternas, obstétricas, pediátricas e socioeconômicas. E é caracterizada pela infecção do vírus de mãe para filho que pode ocorrer durante a gestação, parto ou puerpério, ou no período de amamentação com diferentes chances de transmissão em cada um desses momentos (Siqueira et al., 2020).

Até ao ano de 2016, aproximadamente 2,1 milhões de crianças de até 15 anos viviam com HIV em todo o mundo, sendo a transmissão vertical responsável por mais de $90 \%$ desses casos (UNAIDS, 2017). E na África Subsaariana, mulheres e meninas representaram 59\% de todas as novas infeções por HIV (UNAIDS, 2017); o que significa que ainda teremos novas infeções por transmissão vertical se as medidas profiláticas não serem eficazes nesta região do continente africano.

Segundo Rodrigues et al. (2020), o desejo de ser mãe não diminui na mulher vivendo com HIV, mas fica evidente o medo de transmitir o vírus ao filho. Ressaltase que a mulher que vive com HIV, além de pensar na gravidez, também pensa na saúde do filho durante e após o período gestacional, gerando sentimentos ambíguos, como medo e desejo de conceber na presença do vírus, fazendo com que sua vontade seja prolongada, podendo levar assim a uma gravidez não planejada com alto risco de transmissão vertical.

A prevenção pós-parto da transmissão vertical tornase responsabilidade do cuidador, seja um parente de sangue ou não. O cuidador pode ser empoderado para melhorar a qualidade de vida da criança quando 
é totalmente suportado e orientado por profissionais de saúde. No entanto, quando a orientação e apoio são insuficientes ou inadequados, a criança pode não ter a satisfação nutricional necessária. E para garantir a proteção da vida e reduzir as chances de transmissão vertical, o acesso oportuno ao tratamento profilático e antirretroviral é imperativo (Bick et al., 2017).

\section{Promoção da saúde da mulher HIV positivo}

A promoção da saúde possui um papel importante nesta discussão, à medida que pode buscar estratégias e políticas para evitar a exposição a fatores de risco à Transmissão Vertical no contexto do HIV.

Quanto ao cuidado de mulheres vivendo com HIV no ciclo gravídico e em amamentação, estudo de Cartaxo (2013) aponta que estas vivências podem ser especialmente desafiadoras para as mulheres e permeadas por maiores preocupações com relação a TARV e a possibilidade de Transmissão Vertical (Vescovi et al., 2016).

As indagações sobre como podem ser abordadas a sexualidade e prevenção da Transmissão Vertical do HIV envolvem múltiplos fatores, como por exemplo, as características socioculturais e históricas da população, e os atores envolvidos no suporte a mulher vivendo com HIV. É de grande importância que, a partir das demandas dos atores envolvidos, como as mulheres, parceiros e profissionais de saúde, sejam feitas as articulações necessárias para a formulação e desenvolvimento de políticas públicas e intervenções atentas a todos os elementos.

Segundo Vescovi et al. (2016), elementos como uma assistência pré-natal esclarecedora, a identificação de vulnerabilidades e fatores de risco, o fortalecimento das redes de apoio da mulher, e o devido acolhimento e suporte técnico, informacional e emocional são imprescindíveis na atenção a mulher HIV positivo gestante ou em amamentação. Os estudos de Mann e Monteiro (2018) corroboram ao apresentar a grande importância da identificação e compreensão de fatores de risco, assim como de condições e variáveis que podem estar associadas a questões de riscos em âmbitos biológicos, psíquicos e sociais. É imprescindível ainda que tanto os profissionais como serviços especializados estejam sensibilizados à temática em busca de um acompanhamento mais eficiente e eficaz das mulheres com HIV.
As instituições e profissionais de assistência à saúde possuem um papel fundamental. No entanto, a assistência pode ser comprometida por fatores como o despreparo ao abordar estes temas, a ausência de diretrizes institucionais ou ainda a invisibilidade de aspectos socioculturais, políticos e históricos que perpassam a vivência da sexualidade no contexto do HIV. Quanto mais criarmos espaços para a discussão sobre meios de criar, melhorar e manter esses espaços, menos complicado e mais natural poderá ser lidar com as demandas e dúvidas dos usuários e usuárias de serviços de saúde acerca da sexualidade e da prevenção da transmissão das Infecções Sexualmente Transmissíveis (Mann \& Monteiro, 2018).

\section{Considerações finais}

As mulheres HIV positivas gestantes ou lactantes podem passar por um sofrimento psíquico caracterizado pelo medo de infectar a criança durante a gestação, no parto ou durante a amamentação; referem ainda o receio de partilhar o diagnóstico de HIV positiva enquanto gestantes ou lactantes à família e amigos para evitar a discriminação e estigmatização perante a sociedade que, no geral, ainda não aceita que a mulher HIV positiva também tem o direito de ser mãe como qualquer mulher.

Existe ainda o reconhecimento dos profissionais de saúde sobre a fraca abordagem de assuntos relacionados à saúde mental da mulher HIV positiva nos serviços de atendimento básico de saúde. Dessa forma, é importante pensarmos na promoção da saúde a mulheres HIV positivo gestantes e em amamentação, de forma mais ampla, que considere as discussões em relação a prevenção da Transmissão Vertical do HIV, levando em consideração que o tema perpassa múltiplos fatores, como as características socioculturais e históricas da população, e os atores envolvidos no suporte as mulheres vivendo com HIV.

\section{Contribuições dos autores}

Mazuze BSD escreveu a primeira versão do manuscrito. Borges TDS, Brasil LTMR e Polejack L apoiaram na busca de artigos nas plataformas e revisaram a versão final. Todos os autores revisaram e aprovaram a versão final. 


\section{Conflitos de interesses}

Nenhum conflito financeiro, legal ou político envolvendo terceiros (governo, empresas e fundações privadas, etc.) foi declarado para nenhum aspecto do trabalho submetido (incluindo, mas não se limitando a subvenções e financiamentos, participação em conselho consultivo, desenho de estudo, preparação de manuscrito, análise estatística, etc.).

\section{Referências}

Alvarenga, W. A., Nascimento, L. C., Leal, C. L., Fabbro, M. R. C., Bussadori, J. C. C., Melo, S. S. S., Cartagena-Ramos, D., \& DupasI, D. (2019). Mães vivendo com HIV: a substituição do aleitamento por fórmula láctea infantil. Revista Brasileira de Enfermagem, 72(5), 1217-24. http://dx.doi. org/10.1590/0034-7167-2017-0880

Bastos, R. A., Bellini, N. R., Vieira, C. M., Campos, C. J. G., \& Turato, E. R. (2019). Psychological phases of pregnant women with HIV: a qualitative study in a hospital [Fases psicológicas de mulheres grávidas com HIV: um estudo qualitativo no hospital]. Revista Bioética, 27(2), 281-288. https://doi. org/10.1590/1983-80422019272311

Bellotto, P. C. B., Lopez, L. C., Piccinini, C. A., \& Gonçalves, T. R. (2019). Entre a mulher e a salvação do bebê: experiências de parto de mulheres com HIV. Interface: Comunicação, Saúde, Educação, 23, e180556. https://doi.org/10.1590/ interface.180556

Bick, M. A., Ribeiro, P. L., Ferreira, T., Padoin, S. M. M., \& Paula, C. C. (2017). Fatores intervenientes na alimentação de lactentes expostos à transmissão vertical do HIV: revisão integrativa. Revista Panamericana de Salud Pública, 41, e114. https://doi.org/10.26633/RPSP.2017.114

Cartaxo, C. M. B, Nascimento, C. A. D., Diniz, C. M. M., Brasil, D. R. P. A., \& Silva, I. F. (2013). Gestantes portadoras de HIV/AIDS: aspectos psicológicos sobre a prevenção da transmissão vertical. Estudos Psicologia, 18(3), 419-427. http://dx.doi.org/10.1590/S1413-294X2013000300002

Ethier, K. A., Ickovics, J. R., Fernandez, M. I., Wilson, T. E., Royce, R. A., Koenig, L.J., \& Perinatal Guidelines Evaluation Project Group. (2002). The Perinatal Guidelines Evaluation Project HIV and Pregnancy Study: overview and cohort description [O Projeto de Avaliação de Diretrizes Perinatais HIV e Estudo de Gravidez: visão geral e descrição de coorte]. Public health reports, 117(2), 137-147. https://doi. org/10.1093/phr/117.2.137
Friedrich, L., Menegotto, M., Magdaleno, A. M., \& Silva C. L. (2016) Transmissão vertical do HIV: uma revisão sobre o tema. Boletim Cientifico de Pediatria, 5(3), 81-6. https://www.sprs. com.br/sprs2013/bancoimg/170118174005bcped 0503 a03.pdf

Gonçalves-Germani, A. C. C., \& Aith, F. (2013). Advocacia em promoção da saúde: conceitos, fundamentos e estratégias para a defesa da equidade em saúde. Revista de Direito Sanitário, 14(1), 34-59. https://doi.org/10.11606/issn.23169044.v14i1p34-59

Hoffmann, I. C., Santos, W. M., Silva, C. B., Paula, C. C., Padoin, S. M. M., \& Barros, S. M. O. (2014). Fatores que interferem na transmissão vertical do hiv: revisão integrativa. Enfermagem obstétrica, 1(1), 31-39. http://www.enfo.com. br/ojs/index.php/EnfObst/article/view/8/10

Ingram, D., \& Hutchinson, S. A. (2000). Double binds and the reproductive and mothering experiences of HIVpositive women [Duplo vínculo e as experiências reprodutivas e maternais das mulheres soropositivas]. Qualitative health research, 10(1), 117-132. https://doi. org/10.1177/104973200129118282

Joint United Nations Program on HIV/AIDS. (2016). Get on the FastTrack: the life-cycle approach to HIV [Entre na via rápida: a abordagem do ciclo de vida ao HIV]. https://www.unaids. org/en/resources/documents/2016/get-on-the-fast-track

Joint United Nations Programme on HIV/AIDS. (2017). Latest global and regional statistics on the status of the AIDS epidemic [Últimas estatísticas globais e regionais sobre a situação da epidemia de AIDS]. https://www.unaids.org/en/ resources/documents/2020/UNAIDS_FactSheet

Joint United Nations Programme on HIV/ AIDS. (2018). Miles To Go Closing Gaps Breaking Barriers Righting Injustices [Milhas a percorrer Fechando as brechas que rompem as barreiras que corrigem as injustiças]. http://www.unaids.org/sites/ default/files/media-asset/org/sites/default/files/pub-pdf/ CSE-Global-Review-2015.pdf

Joint United Nations Programme on HIVIAIDS. (2018). Acabando com a epidemia de AIDS entre crianças, adolescentes $e$ mulheres jovens. https://unaids.org.br/2018/04/acabandocom-a-epidemia-de-aids-entre-criancas-adolescentes-emulheres-jovens

Joint United Nations Programme on HIV/AIDS. (2020). Relatório sobre a epidemia HIV. https://bit.ly/3mRvqe3

Kwalombota, M. (2002). The effect of pregnancy in HIV-infected women [O efeito da gravidez em mulheres infectadas pelo HIV]. Aids Care, 14(3), 431-433. https://doi. org/10.1080/09540120220123829 
Mann, C. G., \& Monteiro, S. (2018). Sexualidade e prevenção das IST/aids no cuidado em saúde mental: o olhar e a prática de profissionais no Município do Rio de Janeiro, Brasil. Cadernos de Saúde Pública, 34(7), e00081217. https://doi. org/10.1590/0102-311×00081217

Mindry, D., Wanyenze, R. K., Woldetsadik, M. A., FinoccharioKessler, S., Goggin, K. \& Wagner, G. (2017). Concepção mais segura para casais afetados pelo HIV: considerações estruturais e culturais na prestação de cuidados de concepção mais seguros em Uganda. AIDS and Behavior, 21(8), 2488-96. https://doi.org/10.1007/s10461-017-1816-4

Ministério da Saúde. (2017). Protocolo Clinico e Diretrizes Terapêuticas para o manejo da infeção pelo HIV em crianças e adolescentes. Secretaria de Vigilância em Saúde, Departamento de Vigilância, Prevenção e Controle das Infeções Sexualmente Transmissíveis, do HIV/Aids e das Hepatites Virais. http://www.aids.gov.br/pt-br/pub/2017/ protocolo-clinico-e-diretrizes-terapeuticas-para-manejoda-infeccao-pelo-hiv-em-criancas-e

Ministério da Saúde. (2019). Protocolo Clínico e Diretrizes Terapêuticas para Prevenção da Transmissão Vertical do HIV, Sífilis e Hepatites Virais. Secretaria de Vigilância em Saúde. Departamento de Doenças de Condições Crônicas e Infecções Sexualmente Transmissíveis. http://www. aids.gov.br/pt-br/pub/2015/protocolo-clinico-e-diretrizesterapeuticas-para-prevencao-da-transmissao-vertical-dehiv

Ministério da Saúde de Moçambique. (2019). Inquérito de Indicadores de Imunização, Malária e HIVISIDA em Moçambique (IMASIDA): Relatório Suplementar Incorporado os Resultados de Biomarcadores de Antirretrovirais. https:// www.dhsprogram.com/pubs/pdf/AIS12/AIS12_SP.pdf

Organização Pan-Americana de Saúde/ Organização Mundial da Saúde. (2021). HIV/aids. https://www.paho.org/pt/topicos/ hivaids

Padoin, S. M. M., Souza, Í. E. O., \& Paula, C. C. (2010). Cotidianidade da mulher que tem HIV/AIDS: modo de ser diante da (im)possibilidade de amamentar. Revista Gaúcha de Enfermagem, 31(1), 77-83. https://doi.org/10.1590/ S1983-14472010000100011

Pinto, M. D., Maia, G. N., Pereira, M. D., \& Levandowski, D. C. (2017). Mães adolescentes que vivem com o HIV: uma investigação qualitativa sobre a "Constelação da Maternidade". Psicologia clínica, 29(3), 381-401. http://pepsic.bvsalud.org/scielo.php?script=sci abstract\&pid=S0103-56652017000300003\&lng=pt\&nrm= iso
Rodrigues, J. P., Chaves, L. S., Valois, R. C., Carvalho, D. S., Nascimento, M. H. M., Siqueira, L. S., Oliveira, M. F. V., \& Panarra, B. A. C. S. (2020). Mulheres com HIV: Percepção sobre uma futura gestação. Revista de Enfermagem UFPE On line, 14, e244053. https://doi.org/10.5205/19818963.2020 .244053

Rodriguez, M. J. H., Santos, E. K. A., Meirelles, B. H. S., Frigo, J., \& Rodriguez, A. H. (2014). Perceção da mulher HIV positivo acerca do cuidado pré-natal, parto e puerpério: revisão integrativa. Revista de enfermagem UFPE On line., 8(10), 3492-501. https://periodicos.ufpe.br/revistas/ revistaenfermagem/article/download/10082/10528

Silva, C. B., Motta M. G. C., \& Bellenzani, R. (2019). Maternidade e HIV: desejo reprodutivo, sentimentos ambivalentes e cuidado (não) ofertado. Revista Brasileira de Enfermagem, 72(5), 1378-88. https://doi.org/10.1590/0034-7167-2018$\underline{0063}$

Silva, C. B., Motta, M. G. C., \& Bellenzani, R. (2020). Vivência da gestação e da maternidade por adolescentes/ jovens que nasceram infectadas pelo HIV. Revista Brasileira de Enfermagem, 73(supl 4), e20190405. http://dx.doi. org/10.1590/0034-7167-2019-0405

Siqueira, P. G. B., Miranda, G. M. D., Souza, W. V., Silva, G. A. P., \& Mendes, A. C. G. (2020). Análise hierarquizada dos determinantes da transmissão vertical do HIV: um estudo de caso-controle. Revista Brasileira Saúde Materno Infantil, 20(4), 985-995. https://doi.org/10.1590/180693042020000400005

Sociedade Brasileira de Pediatria. (2017). Guia Prático de Atualização: Doenças Maternas Infecciosas e Amamentação. Departamento Científico de Aleitamento Materno. https:// www.sbp.com.br/fileadmin/user upload/Aleitamento DoencMat_Infec_e_Amam.pdf

Vescovi, G., Castoldi. L., Pereira, M. D. \& Levandowski, D. C. (2016). Fatores de risco para a maternidade entre adolescentes vivendo com HIV. Psicologia Em Estudo, 21(1), 41-52. https://doi.org/10.4025/psicolestud.v21i1.28124

Zihlmann K. F., \& Alvarenga, A. T. (2015). Que desejo é esse? Decisões reprodutivas entre mulheres vivendo com HIV/Aids sob o olhar da psicanálise. Saúde e Sociedade, 24(2), 633-645. https://doi.org/10.1590/S010412902015000200019 